\title{
MEMS-based lidar for autonomous driving
}

\author{
H.W. Yoo, N. Druml, D. Brunner, C. Schwarzl, T. Thurner, M. Hennecke, G. Schitter OVE
}

\begin{abstract}
Lidar, the acronym of light detection and ranging, has received much attention for the automotive industry as a key component for high level automated driving systems due to their high resolution and highly accurate 3D imaging of the surroundings under various weather conditions. However, the price and resolution of lidar sensors still do not meet the target values for the automotive market to be accepted as a basic sensor for ensuring safe autonomous driving. Recent work has focused on MEMS scanning mirrors as a potential solution for affordable long range lidar systems. This paper discusses current developments and research on MEMS-based lidars. The LiDCAR project is introduced for bringing precise and reliable MEMS-based lidars to enable safe and reliable autonomous driving. As a part of development in this project, a test bench for the characterization and performance evaluation of MEMS mirror is introduced. A recently developed MEMS-based lidar will be evaluated by various levels of tests including field tests based on realistic scenarios, aiming for safe and reliable autonomous driving in future automotive industry.
\end{abstract}

Keywords: lidar; MEMS scanning mirror; autonomous driving; metrology platform

\section{MEMS-basiertes Lidar für autonomes Fahren.}

Lidar, ein Akronym für Light Detection And Ranging, erhielt als Schlüsselkomponente für autonome Fahrsysteme in der Automobilindustrie viel Aufmerksamkeit, da es hochauflösende und hochgenaue 3D-Bilder der Umgebung bei verschiedensten Wetterbedingungen liefert. Derzeit entsprechen die Preise und die Auflösung der kommerziell verfügbaren Lidar-Sensoren jedoch noch nicht den Zielanforderungen, um als Basissensor zur Gewährleistung der Sicherheit während des autonomen Fahrens akzeptiert zu werden. MEMS-basierte Scan-Spiegel waren als potentielle Lösung für ein leistbares weitreichendes Lidar-System Fokus einer kürzlich durchgeführten Arbeit. Diese Arbeit erörtert die derzeitige Entwicklung und Forschung von MEMS-basierten Lidar-Systemen und stellt das LiDCAR-Projekt vor, dessen Ziel es ist, ein präzises und verlässliches MEMS-basiertes Lidar-System für autonomes Fahren zu entwickeln. Als Teil-Entwicklung dieses Projekts wird ein Prüfstand zur Charakterisierung und Evaluierung von MEMS Scan-Spiegeln vorgestellt. Das kürzlich entwickelte MEMS-basierte Lidar-System wird auf verschiedenen Testebenen evaluiert, inklusive Feldtests, basierend auf realistischen Szenarien, mit dem Ziel, in Zukunft sicheres und verlässliches autonomes Fahren zu gewährleisten.

Schlüsse/wörter: Lidar; MEMS Scan-Spiegel; autonomes Fahren; Metrologie-Plattform

\section{Introduction}

Lidar is the acronym of light detection and ranging, in an analogy to radar. The basic structure of any pulsed time of flight (TOF) lidar can be described as follows (see Fig. 1): A laser module generates a short laser pulse and the light hits the target object. Then the receiver detects the reflected pulse from the target. From the elapsed time from the transmitted pulse to the received pulse, a data acquisition unit calculates the distance.

Since its first use for highly accurate lunar ranging, many lidar systems have been developed for various applications as a high resolution and highly accurate measurement technique, e.g. for earth surface measurements [1], wind speed measurements [2], building construction operations [3], mining industry [4], forestry [5], and robotics [6]

Recently lidar sensors have received much attention for the automotive industry as a key component for high level automated driving systems [7-9]. Compared to other 3D sensing techniques, such as stereo cameras and radar, lidar sensors can provide high resolution and highly accurate 3D measurements of the surroundings under various weather conditions [10]. The expected function from automotive lidar sensors is to ensure reliable and safe automated driving such as collision detection, blind spot monitoring, object and pedestrian recognition [11, 12], terrain mapping [13]. Currently Google [14], BMW [15], Volvo [16] and other autonomous car developers include lidar sensors in their development to ensure safe driving [17].
The commercially available lidar sensors can be categorized into two types according to the maximum range and the resolution of the system: short range and long range lidar sensors. The short range lidar sensors usually measure less than $50 \mathrm{~m}$ distance with a narrow angle for forward collision warning and blind spot detection [18]. They usually implement a flash lidar structure, shown in Fig. 1. In a flash lidar, the transmitter illuminates the whole scene and an array of detectors measures the distance of each pixel of the image. Continental (Hanover, Germany) and LeddarTech (Quebec City, Canada) provide affordable flash lidar sensors to the market [19, 20]. Short range lidar sensors are low-priced due to the simplicity of the setup, but the measurement distance is fairly short since the light intensity from the transmitter is dispersed with a relatively large angle, and is also limited by eye-safety considerations [21]. In con-

Yoo, Han Woong, Automation and Control Institute, TU Wien, Gußhausstraße 27-29, 1040 Vienna, Austria (E-mail: yoo@acin.tuwien.ac.at); Druml, Norbert, Design Center Graz, Infineon Technologies Austria AG, Babenbergerstraße 10, 8010 Graz, Austria; Brunner, David, Automation and Control Institute, TU Wien, Gußhausstraße 27-29, 1040 Vienna, Austria; Schwarzl, Christian, Virtual Vehicle Research Center, Inffeldgasse 21a, 8010 Graz, Austria; Thurner, Thomas, Design Center Graz, Infineon Technologies Austria AG, Babenbergerstraße 10, 8010 Graz, Austria; Hennecke, Marcus, Design Center Graz, Infineon Technologies Austria AG, Babenbergerstraße 10, 8010 Graz, Austria; Schitter, Georg, Automation and Control Institute, TU Wien, Gußhausstraße 27-29, 1040 Vienna, Austria 

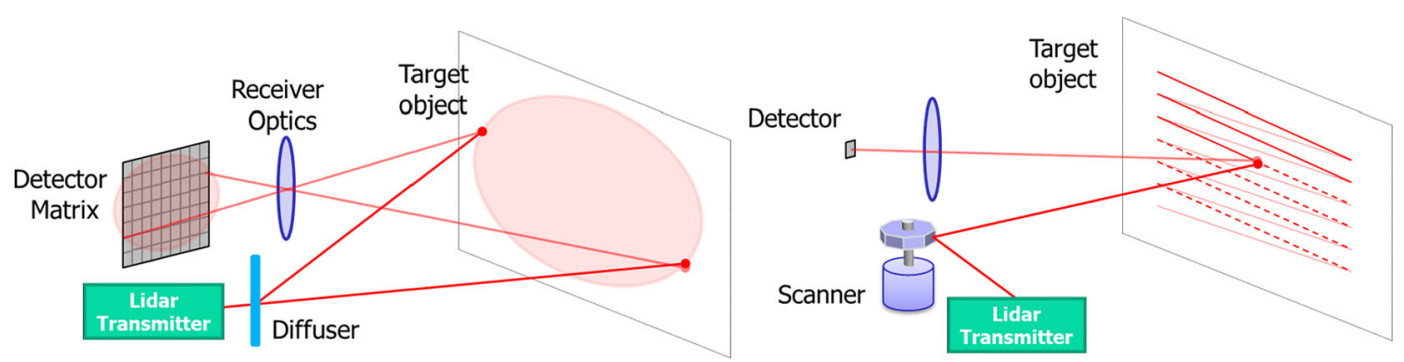

Fig. 1. Structure of lidar system: flash lidar (left) and scanning lidar (right)

trast, scanning lidar sensors, also shown in Fig. 1, focus their light on a very small area, making them suitable for long distance and high resolution imaging. A scanning lidar sensor diverts a collimated beam to the target and illuminates a small fraction of the target object at one shot. Then the reflected light from the small area of the target object is focused on the detector, recording a pixel. By moving the beam position, the distance of the next pixel is measured and a $3 D$ image of the entire scene can be generated. Currently Velodyne (Morgan Hill, CA, US) and Quanergy (Sunnyvale, CA, US) provide entire view $\left(360^{\circ}\right)$ lidars in the market and Ibeo (Hamburg, Germany) provides lidars with a full front range $\left(145^{\circ}\right)$ [22-24]. Scanning lidars can provide rich 3D maps of the surroundings, which allows further analysis such as identification and avoidance of objects and pedestrians.

The currently popular scanning solution in the automotive lidar market is the rotating scanner $[7,25]$. The rotating scanner is currently most popular for many commercial lidar sensors because it provides straight and parallel scan lines with a uniform scanning speed over a wide field of view. Furthermore, it provides less stress and low vibrations on the mirror surface [26]. As a drawback, it is generally bulky and less scalable due to the limiting duty cycle by the edges between facets for polygon mirrors [27]. In addition, a large inertia of the rotating module limits the frame rate of lidar sensors and requires rather high power consumption.

As an alternative solution, optical phase arrays and microelectromechanical system (MEMS) mirrors receive much attention as promising solutions for affordable lidars $[18,28]$ and compete with each other for the future automotive lidar market. An optical phase array (OPA) is a solid-state device that steers the beam by microstructured waveguides. Since there is no mechanical moving part, OPA allows extremely high scanning speed over $100 \mathrm{kHz}$ over large angles. However the insertion loss of the laser power is a drawback $[29,30]$. As a developing technology with high potential, the interests on OPA for automotive lidar is growing in academia and industry even though OPAs are still not proven for long range lidar yet. Microelectromechanical systems (MEMS) mirrors carry a high potential as a future automotive lidar solution since both the form factor and the cost in a large volume can be drastically reduced. The lidar manufactures are keen to test these two promising technologies. Quanergy announced a solid state lidar system (S3) based on optical phase array (OPA) scanner at the Consumer Electronics Show (CES) 2016, which has $120^{\circ}$ optical scanning angle for horizontal and vertical direction and with $0.05^{\circ}$ resolution [31]. Innoviz (Kefar Sava, Israel) also released a MEMS-based solid state lidar (InnovizPro), which allows ranging up to $150 \mathrm{~m}$ distance with a $73^{\circ} \times 20^{\circ}$ field of view (FOV) with $0.15^{\circ}$ horizontal resolution [32]

This work discusses the current research on MEMS-based lidar sensors and introduces a MEMS-based lidar sensor. In Sect. 2, the current state of the art about MEMS-based lidar sensors is reviewed.
A MEMS-based lidar approach in LiDcAR project is introduced and its challenges are elaborated in Sect. 3. A MEMS test bench to evaluate MEMS mirror for lidar application is presented in Sect. 4. Verification and validation of automotive functions using lidar sensors are explained in Sect. 5. Then Sect. 6 concludes the paper.

\section{Review on current development of MEMS-based lidar}

MEMS-based scanning mirrors have recently received much interest for their use in automotive lidar applications as a light-weight, compact and low power consumption scanning solution. Several MEMSbased lidar sensors have already been developed in various application such as space applications [33, 34], robotics [35, 36] and wind velocimetry [37], showing the feasibility of the technology. For automotive applications, Hoffman et al. developed a $360^{\circ}$ scanning 2D MEMS scanner lidar with $60^{\circ}$ of azimuth angle [38] in the EU project of Minifaros [39]. Stann et al. also developed a short range lidar sensor for the small unmanned ground vehicle (UGV) and air vehicle (UAV), whose range is up to $160 \mathrm{~m}$ with a $42^{\circ} \times 21^{\circ} \mathrm{FOV}$ and $6 \mathrm{fps}$ [40]. Ito et al. developed MEMS-based lidar with single photon CMOS focal plane detectors, which can measure distances up to $25 \mathrm{~m}$ with a $45^{\circ} \times 11^{\circ} \mathrm{FOV}$ [41]. MEMS lidar sensors are also used for testing of the lidar technique itself, e.g. digital modulation [42] and optical code division multiple access (OCDMA) [43].

MEMS scanning mirrors can be mainly categorized into resonant MEMS mirrors and non-resonant MEMS mirror according to the operating frequency with respect to their mechanical mode [44]. The resonant MEMS mirrors provide a large scan angle at a high frequency and a relatively simple control design while the scan trajectory is sine-like, i.e. non-uniform scan speed. In addition, MEMS scanner as a nonlinear oscillator can cause softening and or stiffening, limiting the operation frequencies [45]. Non-resonant MEMS mirrors, also called quasi-static MEMS mirrors, provide a large degree of freedom in the trajectory design. Although rather complex controller is required to keep the scan quality, desirable scanning trajectories such as triangular or saw tooth scanning with constant scan speed at large scan ranges can be generated by an appropriate controller design $[46,47]$. Quasi-static MEMS mirrors, however, usually have a smaller scanning angle compared to the resonant MEMS mirrors. To enlarge the scan angle, an additional optics and lens are used $[40,46,48,49]$. An immersion of the MEMS mirror package also increases the scan angle by Snell's window effect with additional damping as a tradeoff [50].

Actuation and scanning principles get diversified, looking for the best technique in the future lidar market. The most common actuation principle is currently the electrostatic comb drive while electromagnetic actuation [51] and piezoelectric actuation [52] have also been reported for lidar applications. Not only flat mirror based scanning, a 1D refractive MEMS scanner has been developed for a $\pm 5^{\circ}$ optical scan angle based on the rotation of a convex lens with comb 
drive. A commercially available digital micro mirror device (DMD) has been applied for a scanning lidar sensor as a programmable grating [53]. Wang et al. developed a new concept of optical phase array based on MEMS approach using vertically actuating comb drives, which can steer the laser beam $\pm 11^{\circ}$ [54].

The figure of merit (FoM) for the MEMS mirror for automotive lidar is different from a typical MEMS mirror for other applications. A FoM for MEMS mirrors is proposed for pico-projector application, which is the product of the scan angle, mirror size, and resonant frequency (i.e. scanning frequency), which defines the resolution of the projector [44]. For long range lidar, scan angle and mirror size are accepted as important parameters. Especially for the bistatic lidar structure, a larger aperture at receiver is important to ensure enough signal to noise ratio (SNR) for long distance detection. Sander et al. propose a dedicated MEMS mirror structure, which consists of a resonant MEMS mirror for the transmitter and 14 identical MEMS mirror for the receiver, operating in a synchronized manner [55]. Besides the mirror size, robustness to the harsh environment conditions such as extremely high or low temperature and large vibrations should be considered [56]. A trade-off between the mirror size and the vibration rejection performance is discussed while an immersion medium in the MEMS mirror package is reported to provide enhanced robustness against external vibrations and shocks [57].

\section{LiDCAR project: evaluation of MEMS-based lidar}

The LiDCAR project, which is a collaborative (Infineon Technologies Austria AG, TU Wien - Automation and Control Institute (ACIN), and the Virtual Vehicle Research Center) FFG funded research project, aims to explore and to assess the two most promising lidar technologies in order to pave the way for the long sought automotivequalified, long-range, robust, and low-cost Lidar solution. This will be accomplished in a three-step process:

1. LiDcAR's first step is to perform a comprehensive laboratorybased evaluation and comparison of the most promising technologies (such as MEMS mirror, rotating polygon mirror, optical phased array, flash Lidar, and fixed multi-beam). Based on the identified strengths and weaknesses, the two most promising technologies are selected and two experimental prototypes are realized

2. The second step of LiDcAR is to research and develop methodologies that will enable essential lidar-metrology and verification methodologies. This effort will pave the way towards a proper lidar evaluation system and will thus enable the path towards an automotive-qualified lidar system.

3. In a third step, in tight collaboration with ALP.Lab, the two experimental lidar prototypes will be integrated into an autonomous test vehicles. Extensive in-field tests at Styria's autonomous driving test-region will provide valuable data that will be analyzed and assessed according to the developed lidar-metrology and verification methodologies. The gained results and insights will finally pave the way towards the future automotive-qualified and low-cost key lidar technology made in Austria.

The LiDCAR project and its consortium identified the microscanning 1D MEMS-mirror lidar as one of the currently most promising lidar technologies. Therefore, the consortium is currently intensively working on a very first MEMS-based lidar prototype. Its fundamental scanning concept is depicted in Fig. 2. A 1D MEMS mirror oscillates and deflects a laser pulse into the scenery. It is controlled and actuated by a dedicated driving circuitry. Laser pulses reflected off objects in the scenery are focused by optics and detected by an array of photo diodes. The received analog signals are amplified via transimpedance amplifiers and high-speed analog-to-digital and time-to-digital conversions are carried out. Finally, a 3D point cloud can be computed and forwarded to a central sensor fusion ECU. In this particular approach to lidar sensors the MEMS mirror oscillates about only one axis, horizontally scanning the scenery left-right. At the same time, the laser beam fans out and paints a vertical line in the distance, resulting in a full scan of the scenery. This unique approach results in a number of key features: The horizontal resolution of the system is entirely determined by the timing accuracy of the MEMS driving circuit and can reach $0.1^{\circ}$ or better. Since all angles in the vertical direction are scanned in parallel with the same laser pulse, the scan speed is very high. With the laser, light focused into one thin line the pulse energy is concentrated in a small area enabling long range distance measurements. And finally, a MEMS mirror with only one axis is far less complex to manufacture and to operate than a two axis mirror and it is also far more robust to vibration and shock.

A major LiDCAR result achieved so far is the realization of a first feasibility study. This feasibility study includes the MEMS mirror and a very first version of a future MEMS driver circuitry, as depicted in Fig. 3. Together both components form the central and most crucial part in the micro-scanning 1D MEMS-mirror lidar concept. Preliminary results already proved successfully the feasibility of this lidar concept. Furthermore, this platform enables rapid technology explorations and rapid prototyping, which is crucial in the light of system complexity and the fast-paced lidar research arena.

\section{Test bench development for MEMS scanning mirror}

The characterization of MEMS scanning mirrors is crucial in the development of the MEMS mirror, in order to measure and evaluate the performance and reliability of the developed mirror prototypes in order to ensure to meet all requirements needed to be use in the automotive environment as essential part of automotive lidar systems. In our 1D scanning lidar approach the MEMS mirror is used to steer the laser beam in a deterministic and reproducible manner. The characterization of such MEMS mirror needs the real-time measurement of the mirror deflection together with actuation voltage signal and the implemented current sensing approach, but with guaranteed accuracy and resolution in a calibrated measurement setup. The characterization environment thus should provide the scanning trajectories in degree within a required measurement uncertainty, resolution and possibly appearing distortion. Repeatability and reproducibility of the developed setups should considered with included geometric calibration procedures, to correct for all relevant systematic error contributors to the scan trajectory measurement. The signal quality from all sensor readouts should be high to meet the required challenging resolution and measurement uncertainty requirements, regardless of noise and external disturbances such as electromagnetic interference (EMI) from high voltage operation of the MEMS scanning mirror. For such a purpose, a test bench for MEMS scanning mirrors has been developed and implemented

Figure 4 describes schematics of the mirror test bench. The test bench mainly consists of a laser unit, a position sensitive device unit, and adjustable mounts for the direct measurement of the mirror deflection, including calibration and alignment. The laser unit consists of an adjustable fiber collimator from an external fiber laser sources. The laser is focused via the central region of the MEMS mirror onto a position sensitive device (PSD) (S5991-01, 2D PSD, Hamamatsu). This PSD element allows for real-time high bandwidth tracking of the reflected laser beam position on the active PSD area. For the accuracy of geometrical alignment, the laser collimator is attached on a 5 DOF mount, correcting position and direction of the laser 


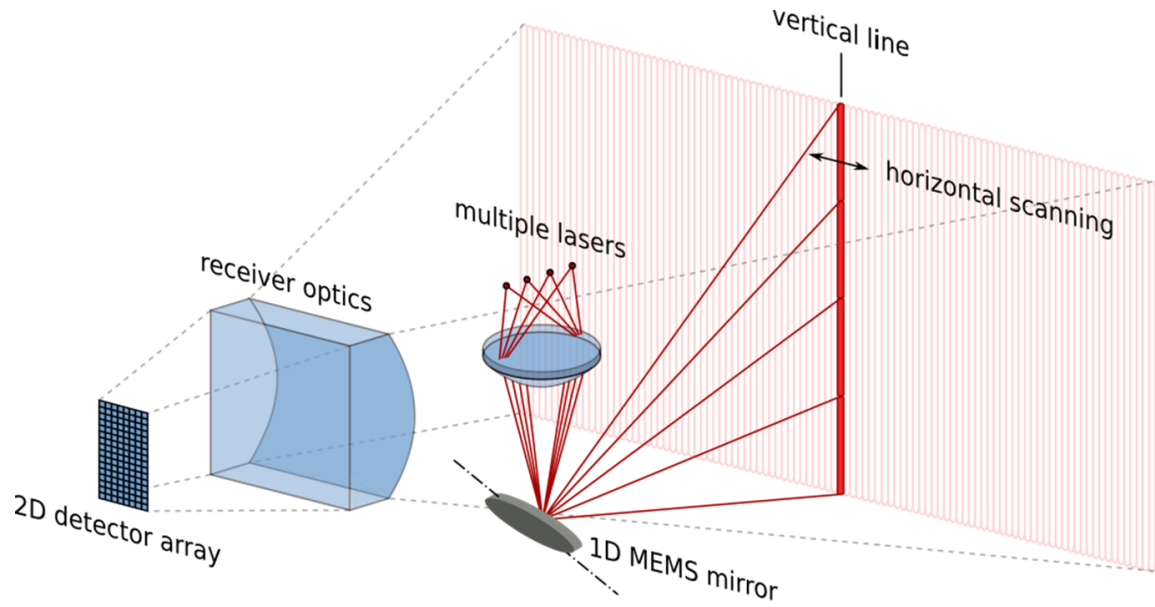

Fig. 2. Working principle of the micro-scanning 1D MEMS-mirror lidar approach

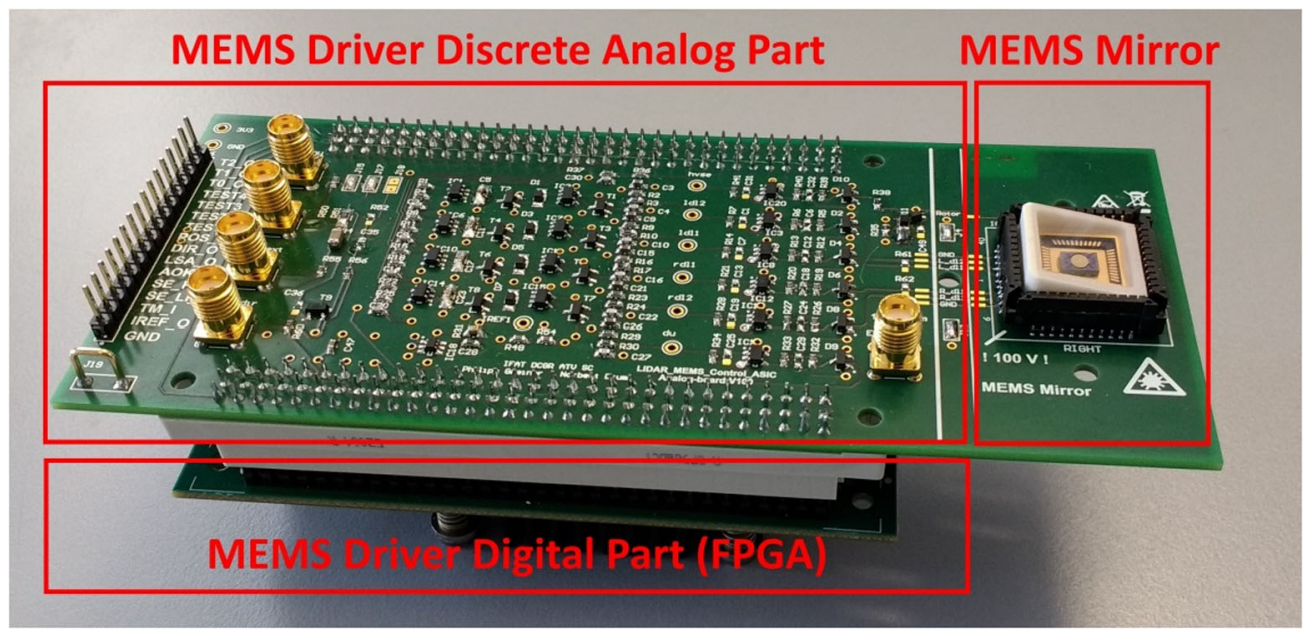

Fig. 3. MEMS mirror and driver feasibility study

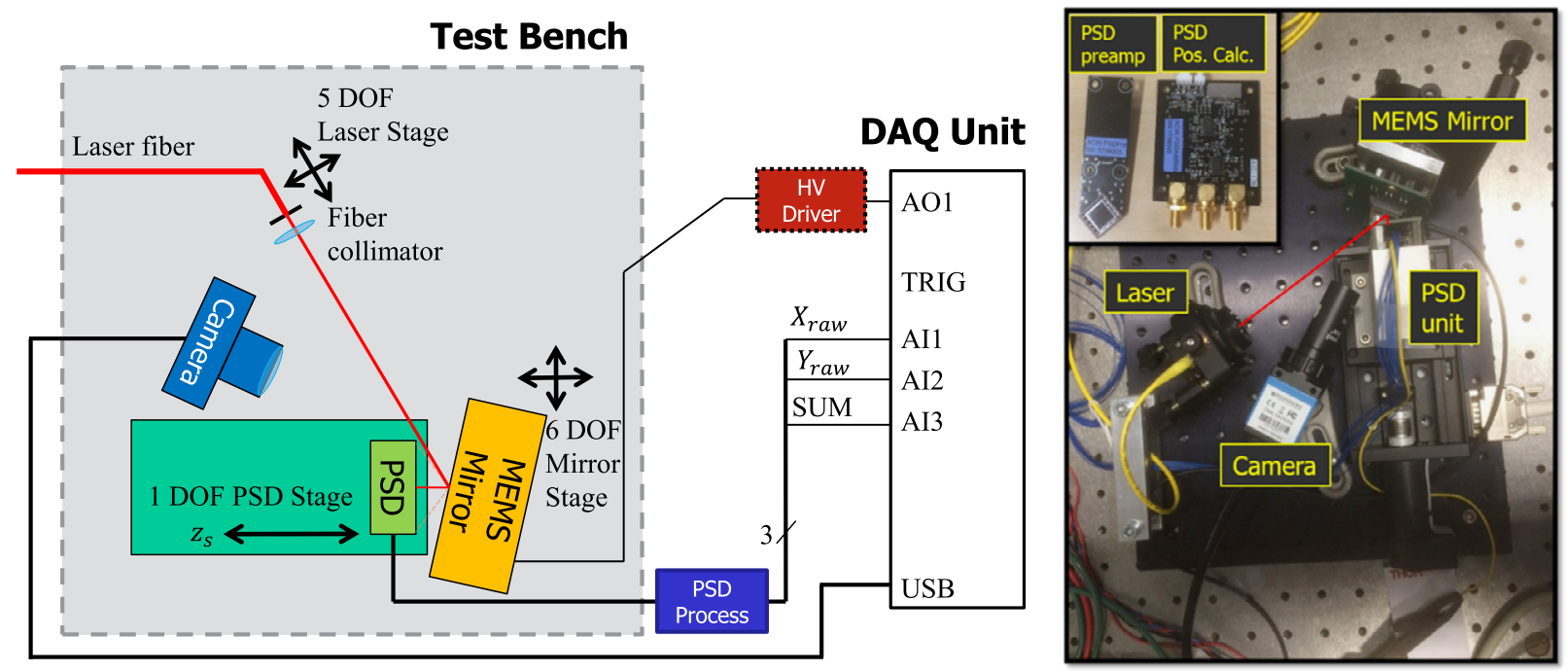

Fig. 4. Schematics and a picture of the test bench. The laser is equipped with a 5 DOF optics mount, the mirror is attached with a 6 DOF mirror mount, and the PSD is mounted on a 1 DOF motorized stage for the alignment 
beam. The MEMS scanning mirror is placed on a 6 DOF mount to correct all possible device orientation and tilt errors of the MEMS mirror in the given package prototype. Proper positioning of the MEMS mirror after replacement and inclusion in the setup. The position error during the mirror exchange procedure is measured by a high resolution digital camera with attached telecentric objective. An image processing software provides the position and direction error of the mirror, allowing an intuitive support for proper manual adjustment of the MEMS mirror mount. The PSD unit is attached on a fixture mounted on a 1D motorized linear translation stage, which enables the measurement and of the geometrical correction factors as needed by the geometrical calibration process. The enable maximum range for mirror deflection sensing the PSD is tilted by $45^{\circ}$ on the mount, having the scan trajectory aligned with the diagonal of the quadratic-shaped active PSD area. On the electrical side the PSD unit is connected to a dedicated preamplifier module with transimpedance amplifiers (TIA) and a following analog processing module. All analog processing circuits have optimized for robustness against the EMI generated by the high voltage operation of the closely located MEMS mirror.

Systematic errors from the alignment of the individual test bench components are the major contributors to the achievable accuracy of the mirror deflection measurement. One of the major errors source is the distance between the PSD and the MEMS mirror, which needs to be determined with an uncertainty of less than tens of micrometers to ensure the required accuracy in the measurement. A dedicated calibration process has been developed to estimate the needed geometrical correction factors for enabling a highly accurate mirror deflection measurement.

Figure 5 provides an example of a measurement result from the developed test bench, showing a typical response curve as one of the main characteristics of a MEMS mirror prototype operated in open loop. The diagram shows the evolution of MEMS mirror deflection angle amplitude over frequency when starting the mirror and operating up to the high angle operation point - in this case at $15^{\circ}$ mechanical angle amplitude.

By providing accurate mirror angle data the developed setup including the geometrical calibration procedure - allows for reliable high accuracy characterization of MEMS scanning mirrors with high repeatability and reproducibility

\section{Verification and validation of MEMS-based lidar for autonomous driving}

Before a new vehicle function can go into serial production a thorough verification of its functional correctness is necessary not only to ensure customer satisfaction, but also to avoid safety threats to the vehicle inhabitants or other traffic participants in case of safety critical functions. This is achieved by performing tests at different test levels, where the developed function parts are integrated and tested using real or simulated stimulations. With increasing test level the test environments become closer to reality, where in the lowest level the function is tested using purely simulated inputs and in the highest level only real sensor data is used obtained by during test drives on public roads.

An example of the main test levels considered in the automotive industry is shown in Fig. 6, where also a commonly used test environment is depicted for each test level. The shown test levels reflect the integration steps of the developed function into the vehicle, where in level one and two its hardware and software parts are tested and in level three to five the function is tested in combination with other vehicle functions.

Since testing at test levels three to five is very costly and time consuming, the LiDCAR consortium cooperates tightly with ALP.Lab

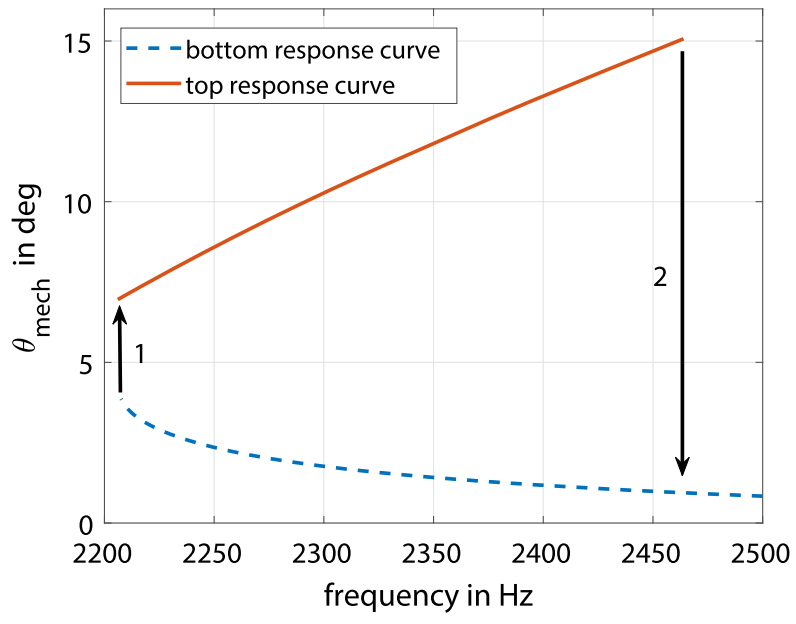

Fig. 5. Frequency response of the MEMS mirror, measured by the developed test bench. The response curve of the MEMS mirror consists of top response curve (red solid line) and bottom response curve (blue dashed line). When sweeping down the excitation frequency on the mirror along the bottom response curve, a jump (labeled 1, with an up arrow) to the top response curve at higher angle amplitudes is observed. The scan angle increases further by an following up sweep after the jump, following the top response curve. At a certain driving frequency, the angle amplitude falls back (labeled 2, with a down arrow) to the bottom response curve (Color figure online)

(Austrian Light Vehicle Proving Region for Automated Driving) in order to get access to wide range of test hardware, proving grounds and permissions for public road tests. In particular, the access to large number of test tracks from AVL, Magna Steyr, Zentrum am Berg, Testregion Lungau, or the Redbull race track in Spielberg allows the simulation of a wide range of environment conditions and driving situations. On these test tracks automated robots will be used to move objects like pedestrians, cars and cyclists into the field of view of the lidar sensors. This approach allows the execution of tests under a wide range of weather conditions with known objects and their position, which ensures the reproducibility of the test results and ensures that the sensor measurement data can be automatically verified for correctness. The simulation environments provided by ALP.Lab can also be used to specify the tests performed on the proving ground, increasing efficiency during test development.

In addition, ALP.Lab provides highly accurate sensor systems, which can be mounted on the test vehicle, allowing a performance assessment of the used lidar sensors. Moreover, ALP.Lab provides a cloud-based data management solution for seamless data acquisition, storage, and automated analysis allowing global access to the obtained measurement data and test results. By using this cloud based storage and analysis framework it is possible to process a large number of test drives within a short time by leveraging the massive parallel computation power provided by such systems. The cloud based storage approach, where each ALP.Lab customer has its own tenant ensuring protection against unauthorized access, is capable of handling many terabyte of data. This large amount of storage is necessary because the different sensors, especially lidar systems, produce measurement data at a very high rate, making the use of latest hard disks infeasible.

\section{Conclusion}

This paper discusses the current developments and research on MEMS-based lidar systems. To ensure safe autonomous driving so- 


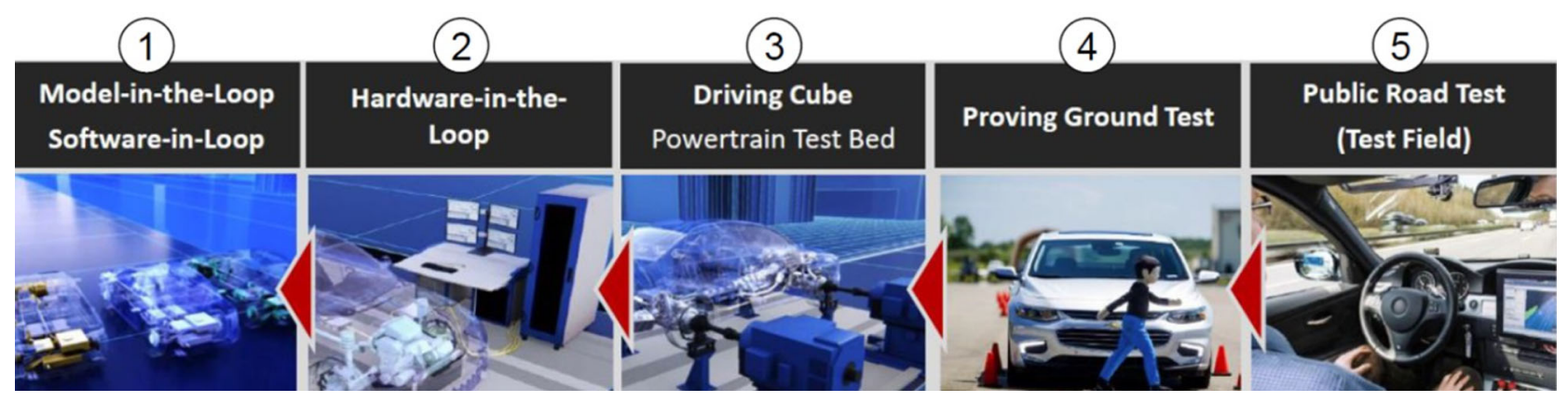

Fig. 6. Test levels ranging from 1 to 5 and their typical test environments used for testing automotive functions

lutions in future mobility, besides 2D cameras and radar sensors an automotive qualified lidar system is seen essential to enable proper perception of the car environment during all driving situations. Stringent requirements on such lidar systems are long range, high resolution and accuracy in distance but also high angular resolution in lateral and vertical direction w.r.t. sensing direction, secured long lifetime in the automotive environment conditions (temperature range, temperature changes, vibration and shock robustness), and, altogether at an acceptable low price. MEMS-based lidar is regarded as one of the most promising solutions for affordable automotive systems, and many rising but also well-established companies as well as research institutions are currently working with high intensity to bring it into the future automotive lidar market.

To cope with the challenging tasks during lidar system development, the LiDCAR project aims in investigation and validation of automotive lidar solutions. The aims and underlying main work packages of the LiDCAR project have been described throughout the paper, targeting in development on characterization environments for lidar components but also lidar systems, and the validation of lidar system prototypes in the automotive environment with the ALP.Lab automated driving test roads. Currently the concept of the feasibility study is successfully finished, resulting in a working MEMS mirror based scan system where a 1D MEMS mirror is interfaced by an FPGA-based evaluation platform. This evaluation platform is based on the current version of a MEMS driver ASIC sensing and control concept - implemented on the FPGA board as a part of the evaluation system. First results have proven suitability for the chosen mirror and driver concept for use in automotive scanning lidar applications. As another part of the project work, a test bench for detailed characterization of scanning MEMS mirrors has been developed. The test bench includes a real-time 2D PSD based optical readout of the mechanical deflection of the MEMS mirror for all operation modes, enabling reproducible high accuracy electromechanical characterization of MEMS mirror devices.

As next steps, the developed test benches will be further optimized and extended towards the robustness evaluation of scanning lidar components and systems. Lidar system prototypes will be realized and these MEMS-based lidar solutions will be evaluated for use in automotive applications, including field tests based on realistic driving scenarios.

\section{Acknowledgements}

Open access funding provided by TU Wien (TUW). This work was supported by Mobilität der Zukunft program of the Austrian Research Promotion Agency (FFG).

Open Access This article is distributed under the terms of the Creative Commons Attribution 4.0 International License (http://creativecommons.org/ licenses/by/4.0/), which permits unrestricted use, distribution, and reproduction in any medium, provided you give appropriate credit to the original author(s) and the source, provide a link to the Creative Commons license, and indicate if changes were made.

\section{References}

1. Glennie, C. L., Carter, W. E., Shrestha, R. L., Dietrich, W. E. (2013): Geodetic imaging with airborne LiDAR: the Earth's surface revealed. Rep. Prog. Phys., 76(8), 086801.

2. Manninen, A. J., O'Connor, E. J., Vakkari, V., Petäjä, T. (2016): A generalised background correction algorithm for a Halo Doppler lidar and its application to data from Finland. Atmos. Meas. Tech., 9, 817-827.

3. Zhang, K., Yan, J., Chen, S.-C. (2006): Automatic construction of building footprints from airborne LIDAR data. IEEE Trans. Geosci. Remote Sens., 44(9), 2523-2533.

4. Schumann, A., Arndt, D., Wiatr, T., Götz, A. E., Hoppe, A. (2011): High-resolution terrestrial laser scanning and 3D modelling of a mineral deposit for extraction management optimisation. Z. Deutsch. Gesellschaft Geowissenschaft., 162(4), 435-442.

5. Kelly, M., Di Tommaso, S. (2015): Mapping forests with Lidar provides flexible, accurate data with many uses. California Agricult., 69(1), 14-20.

6. Gouveia, B. D., Portugal, D., Silva, D. C., Marques, L. (2015): Computation sharing in distributed robotic systems: a case study on SLAM. IEEE Trans. Autom. Sci. Eng., 12(2), 410-422.

7. Lidar: driving the future of autonomous navigation. Frost \& Sullivan, CA, USA, 2016.

8. Rasshofer, R. H., Gresser, K. (2005): Automotive radar and lidar systems for next generation driver assistance function. Adv. Radio Sci., 3, 205-209.

9. Gotzig, H., Geduld, G., (2015): LIDAR-sensorik. In Handbuch Fahrerassistenzsysteme: Grundlagen, Komponenten und Systeme für aktive Sicherheit und Komfort (S. 317334). Berlin: Springer.

10. Laux, T. E., Chen, C.-I. (2014): 3D flash lidar visiton systems for imaging in degraded visual environments. In Degraded visual environments: enhanced, synthetic, and external vision solution. Proc. SPIE (Vol. 9087)

11. Wang, H., Wang, B., Liu, B., Meng, X., Yang, G. (2017): Pedestrian recognition and tracking using 3D LiDAR for autonomous vehicle. Robot. Auton. Syst., 88, 71-78.

12. Himmelsbach, M., Müller, A., Lüttel, T., Wünsche, H.-J. (2008): LIDAR-based 3D object perception. In Proceedings of 1st international workshop on cognition for technical systems.

13. Hata, A., Wolf, D. (2014): Road marking detection using lidar reflective intensity deata and its application to vehicle localization. In IEEE17th international conference on intelligent transportation system, Qingdau, China.

14. Poczter, S. L., Jankovic, L. M. (2014): The Google car: driving toward a better future? J. Business Case Stud., 10(1), 7-14.

15. Chauffeur Inklusive: BMW Active Assist, BMW [Online]. Available: http://www.bmw.com/ [Accessed 3 Feb. 2017].

16. Ross, P. E. (11 May 2016): Volvo's self-driving program will have redundancy for everything. IEEE Spectrum.

17. Ackerman, E. (17 August 2016): Ford and Baidu invest $\$ 150$ million in velodyne for affordable lidar for self-driving cars. IEEE Spectrum.

18. Thakur, R. (2016): Scanning LIDAR in advanced driver assistance systems and beyond. IEEE Cosum. Electron. Mag. 5(3), 48-54.

19. LeddarTech Inc., (2015): Leddar M16 multi-element sensor module.

20. Continental, SRL $1+$ SRL 1C infrrot short range lidar sensor (2009).

21. Toth, C. K. (2009): R\&D of mobile lidar mapping and future trends. In Annual conference ASPRS, Baltimore, Maryland, US.

22. Velodyne (2017): Data sheet: puck real time 3D LiDAR sensor.

23. Quanergy Systems, Inc. (2015): 360 3D LIDAR M8-1 sensor.

24. Ibeo Automotive Systems GmbH (2014): User manual Ibeo ScaLa B2 ${ }^{\circledR}$ laserscanner. 
25. Stone, W. C., Juberts, M., Daglakis, N., Stone, J., Gorman, J. (2004): Performance analysis of next generation LADAR for manufacturing, construction, and mobility. United states department of commerce.

26. Ullrich, A., Pfennigbauer, M., Rieger, P. (2013): How to read your LIDAR spec - a comparison of single-laser-output and multi-laser-output LIDAR instruments. In Riegl laser measurement systems $\mathrm{GmbH}$.

27. Stutz, G. E. (2012): Polygon scanners: components, performance, and design. In G. F. Marshall, G. E. Stutz (Eds.), Handbook of optical and laser scanning (pp. 247-279). Boca Raton: CRC Press.

28. Fersch, T., Weigel, R., Koelpin, A. (2017): Challenges in miniaturized automotive longrange lidar system design. In Three-dimensional imaging, visualization, and display, Anaheim, CA, USA. Proc. SPIE (Vol. 10219).

29. Yaacobi, A., Sun, J., Moresco, M., Leake, G., Coolbaugh, D., Watts, M. R. (2014): Integrated phase array for wide angle beam. Opt. Lett., 39(15), 4575.

30. Sun, J., Timurdogan, E., Yaacobi, A., Hosseini, E. S., Watts, M. R. (2013): Large-scale nanophotonic phase array. Nature, 493, 195-199.

31. Ackerman, E. (7 January 2016): Quanergy announces $\$ 250$ solid-state LIDAR for cars, robots, and more. IEEE Spectrum.

32. Innoviz Technologies (2018): Data sheet of InnovizPro - high performance solid-state LIDAR.

33. Mizuno, T., Mita, M., Kajikawa, Y., Takeyama, N., Ikeda, H., Kawahara, K. (2008): Study of two-dimensional scanning LIDAR for planetary explorer. In Sensors, systems, and next-generation satellites XII.

34. Park, I. H., Jeon, J. A., Nam, J., Nam, S., Lee, J., Park, J. H., Yang, J., Ebisuzaki, T., Kawasaki, Y., Takizawa, Y., Wada, S. (2009): A new LIDAR method using MEMS micromirror array for the JEM-EUSO mission. In Proceedings of the 31st ICRC.

35. Moss, R., Yuan, P., Bai, X., Quesada, E., Sudharsanan, R., Stann, B. L., Dammann, J. F., Giza, M. M., Lawler, W. B. (2012): Low-cost compact MEMS scanning ladar system for robotic applications. In Laser radar technology and applications XVII. Proc. SPIE (Vol. 8379).

36. Sudharsanan, R. (2013): Low cost scanning lidar imager. LIDAR Mag., 3(2).

37. Hu, Q., Pedersen, C., Rodrigo, P. J. (2016): Eye-safe diode laser Doppler lidar with a MEMS beam-scanner. Opt. Express, 24(3), 1934-1942.

38. Hofmann, U., Senger, F., Soerensen, F., Stenchly, V., Jensen, B., Janes, J. Biaxial resonant $7 \mathrm{~mm}$-MEMS mirror for automotive LIDAR application.

39. E. T. P. o. S. S. I. (EPoSS) (2015): European roadmap smart systems for automated driving.

40. Stann, B. L., Dammann, J. F., Giza, M. M. (2016): Progress on MEMS-scanned ladar. In Laser radar technology and applications XXI. Proc. SPIE (Vol. 9832).

41. Ito, K., Niclass, C., Aoyagi, I., Matsubara, H., Soga, M., Kato, S., Maeda, M., Kagami, M. (2013): System design and performance characterization of a MEMS-based laser scanning time-of-flight sensorbased on a 256x64-pixel single-photon imager. IEEE Photonics J., 5(2), 6800114

\section{Authors}

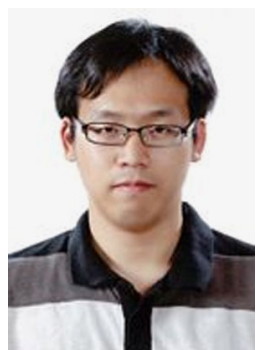

\section{Han Woong Yoo}

was born in Seoul, Republic of Korea. After his bachelor study at Yonsei University, he finished the master study in electrical engineering at Seoul National University in 2007 He worked in Samsung Advanced Institute of Technology (SAIT) and Samsung Electronics co. LTD, semiconductor business for low power digital RF and algorithms for reliability of multi-level non-volatile memories. In 2015 he received a Ph.D. from Delft University of Technology, Netherlands, about adaptive optics and optomechatronics for confocal microscopy. Currently, he is working at the Institute auf Automation an Control (ACIN) at Vienna University of Technology, Austria, as a postdoctoral researcher. His main research interests are optical metrology, precision mechatronics, and biomedical imaging.
42. Giese, T., Janes, J. (2015): 2D MEMS scanning for LIDAR with sub-Nyquist sampling, electronics, and measurement procedure. In Three-dimensional imaging, visualization, and display. Proc. SPIE (Vol. 9495)

43. Kim, G., Park, Y. (2016): LIDAR pulse coding for high resolution range imaging at improved refresh rate. Opt. Express, 24(21), 23810-23828.

44. Holmström, S. T. S., Baran, U., Urey, H. (2014): MEMS laser scanners: a review. J. Microelectromech. Syst., 23(2), 259-275.

45. Agarwal, M., Park, K. K., Candler, R. N., Kim, B., Hopcroft, M. A., Chandorkar, S. A., Jha, C. M., Melamud, R., Kenny, T. W., Murmann, B. (2006): Nonlinear characterization of electrostatic MEMS resonators. In IEEE international frequency control symposium and exposition, Miami, FL, US.

46. Milanović, V., Kasturi, A., Yang, J., Hu, F. (2017): Closed-loop control of gimbal-less MEMS mirrors for increased bandwidth in LiDAR applications. In Laser radar technology and applications, Anaheim, CA, US. Proc. SPIE (Vol. 10191).

47. Milanović, V., Kasturi, A., Yang, J., Su, Y. R., Hu, F. (2018): Iterative learning control (ILC) algorithm for greatly increased bandwidth and linearity of MEMS mirrors in LiDAR and related imaging applications. In MOEMS and miniaturized systems XVII, San Francisco, CA, US. Proc. SPIE (Vol. 10545).

48. Lee, X., Wang, C. (2015): Optical design for uniform scanning in \{MEMS\}-based 3D imaging lidar. Appl. Opt., 54(9), 2219-2223.

49. Yang, B., Zhou, L., Zhang, X., Koppal, S., Xie, H. (2017): A compact MEMS-based wide-angle optical scanner. In 2017 international conference on optical MEMS and nanophotonics

50. Zhang, X., Koppal, S. J., Zhang, R., Zhou, L., Butler, E., Xie, H. (2016): Wide-angle structured light with a scanning MEMS mirror in liquid. Opt. Express, 24(4), 256226.

51. Ataman, Ç., Lani, S., Noell, W., Rooij, N. D. (2013): A dual-axis pointing mirror with moving-magnet actuation. J. Micromech. Microeng., 23(2), 025002.

52. Ye, L., Zhang, G., You, Z. (2017): 5 V compatible two-axis PZT driven MEMS scanning mirror with mechanical leverage structure for miniature LiDAR application. Sensors, 17(3), 521

53. Smith, B., Hellman, B., Gin, A., Espinoza, A., Takashima, Y. (2017): Single chip lidar with discrete beam steering by digital micromirror device. Opt. Express, 25(13), 14732-14745.

54. Wang, Y., Kyoungsik, Y., Wu, M. C. (2016): MEMS optical phased array for LIDAR. In The 21st microoptics conference (MOC), Berkeley, CA, US

55. Sandner, T., Wildenhain, M., Gerwig, C., Schenk, H., Schwarzer, S., Wölfelschneider, H. (2010): Large aperture MEMS scanner module for 3D distance measurement. In MOEMS and miniaturized systems IX. Proc. SPIE (Vol. 7594)

56. Wolter, A., Hsu, S.-T., Schenk, H., Lakner, H. K. (2005): Applications and requirements for MEMS scanner mirrors. In MOEMS and miniaturized systems V, San Jose, CA, US. Proc. SPIE (Vol. 5719)

57. Milanovic, V., Kasturi, A., Yang, J. (2016): Novel fluidic packaging of gimbal-less MEMS mirrors for increased optical resolution and overall performance. In SPIE defense and commercial sensing conference, Baltimore, MD, US.

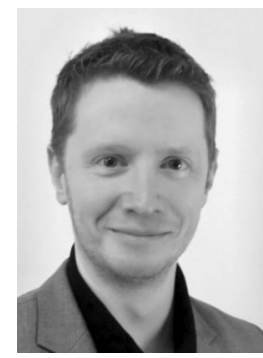

\section{Norbert Drum}

was born in Klagenfurt, Austria in 1980. After being self-employed for ten years in the field of embedded systems development, he received a master's degree in telematics and a doctoral degree in electrical engineering, both from Graz University of Technology in Austria. In 2014, he joined Infineon Technologies Austria AG, where he was leading several industrial research projects (in the fields of embedded systems, sensors, and security) and where he worked as a concept engineer for the next generation sensor chips. His research interests include hardware/software co-design, secure embedded systems, and automotive sensing technology. 


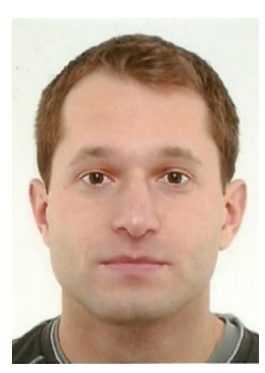

\section{David Brunner}

was born in Waidhofen an der Thaya, Austria in 1990. After working 5 years at Siemens $\mathrm{AG}$ as a working student and 1 year at $\mathrm{Vi}$ enna University of Technology as a student assistant, he received the master's degree in energy engineering and automation technology. In 2017 he started his doctoral studies in electrical engineering at TU Vienna, where he is working on the control concept of a MEMS-based LiDAR system for autonomous driving. His research interests include advanced identification and control concepts, optomechatronic systems and system integration.

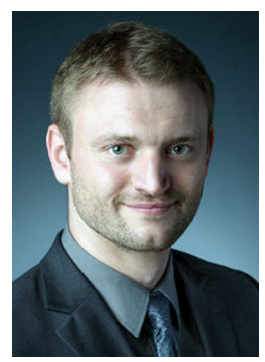

Christian Schwarzl

started his career after finishing the information and computer engineering studies as researcher at the Virtual Vehicle Research Center in Graz, Austria. He began his computer sciences dissertation in parallel, which he finished with honours in 2012. He became Lead Researcher in 2013 and managed the activities in the field of verification and validation. Since 2014 he has been head of the Dependable Systems Group, which primarily focusses on the verification and validation of autonomous vehicles, functional safety and security. Since 2016 he has been active member of the ISO/TC22/SC 32NWG 8 committee for functional safety and contributes to standards like ISO 26262 and ISO PAS 21448 - Safety of the intended functionality.

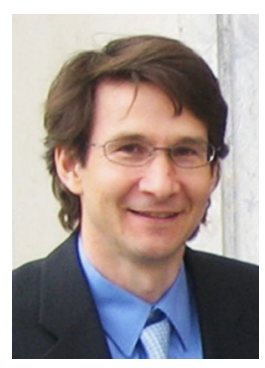

\section{Thomas Thurner}

finished his studies in electrical engineering at the Graz University of Technology in 1999 , focusing on optical metrology and measurement signal processing during the following Ph.D. studies in technical sciences and postdoc academic research at the Institute of Electrical Measurement and Measurements Signal Processing at the Graz University of Technology. From 2008 to 2015 Dr. Thurner was heading the Fatigue Testing Facility at the Graz University of Technology with strong involvement in research on measurement principles for mechanical quantities and with the development and optimization of multi-channel mechanical test control systems. In May 2015 Dr. Thurner joined Infineon Technologies Austria at the Graz Development Center, where he heads the Component Verification group for the Automotive Sense \& Control Graz Department.

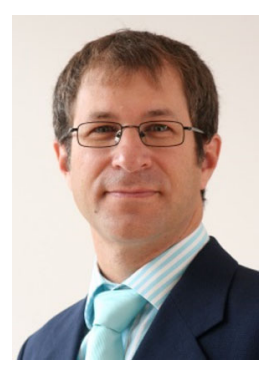

\section{Marcus Hennecke}

studied electrical engineering at the Technische Universität Darmstadt, Germany, completing his studies in 1991 with a diploma degree. In 1992 he graduated from Stanford University with a Master of Science degree in the area of neural networks and continued as doctoral student on the topic of computeraided lip reading. In 1996 he completed his thesis and moved to Ulm in Germany where he worked on an automated spoken language translator at the Daimler-Benz research center until 1998. In the years to come until 2006 he worked on automated speech recognition for automotive dialog systems for the companies Daimler-Benz Aerospace AG, TEMIC Telefunken and Harman Backer as head of the departments algorithms, research and speech recognition, respectively. In 2006 he then moved to Graz, Austria where he headed the business segment Pattern Recognition and Image Processing (PRIP) at the Biometric Center of Siemens up to 2009.

From 2009 to 2016 he worked for EFKON AG as head of the departments Frontend and Machine Vision. Since November 2016 Mr. Hennecke has been heading the Concept Engineering department at the Design Center Graz of Infineon Technologies. In his professional career Mr. Hennecke has initiated and executed various successful national (German and Austrian) as well as European funded projects. He is (co-)author of numerous publications and patents.

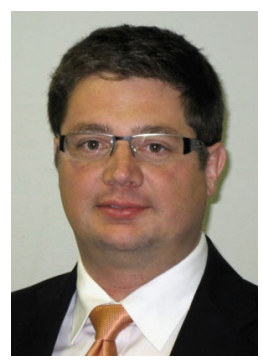

\section{Georg Schitter}

is Professor for Advanced Mechatronic Systems at the Automation and Control Institute (ACIN) of TU Wien. He received a M.Sc. in electrical engineering from TU Graz, Austria (2000) and an M.Sc. and Ph.D. degree from ETH Zurich, Switzerland (2004). His primary research interests are on high-performance mechatronic systems, particularly for applications in the high-tech industry, scientific instrumentation, and mechatronic imaging systems, such as AFM, scanning laser and LIDAR systems, telescope systems, adaptive optics, and lithography systems for semiconductor industry. He received the IFAC Mechatronics best paper award (2008 to 2011) and 2013 IFAC Mechatronics Young Researcher Award, and served as an Associate Editor for IFAC Mechatronics, Control Engineering Practice, and for the IEEE Transactions on Mechatronics. 